\title{
Descripción y diferencias en las variables psicológicas relacionadas con el rendimiento deportivo de triatletas y para-triatletas \\ Description and differences in the psychological variables related to sports performance of triathletes and para-triathletes \\ *Guillermo Gomez-Marcos, **Mauro Sanchez-Sanchez \\ **Universidad Miguel Hernandez de Elche (España), **Universidad Castilla La Mancha
}

\begin{abstract}
Resumen. Tal y como sugieren algunos estudios, las habilidades psicológicas de los para-atletas pueden ser más potentes que las de los atletas, debido a la superación de barreras físicas, comunicativas y económicas, además de traumas. El objetivo de este estudio fue conocer si existían diferencias en las variables psicológicas implicadas en el rendimiento deportivo por la existencia o no de la discapacidad en el triatlón. 18 triatletas y 18 para-triatletas españoles de nivel nacional e internacional participaron en el estudio. Para el estudio fue utilizado el cuestionario Características psicológicas relacionadas con el Rendimiento Deportivo (CPRD). Los resultados obtenidos con la muestra seleccionada de triatletas expertos indican que no existen diferencias $(p<, 05)$ en las variables psicológicas analizadas por el CPRD por la existencia o no de la discapacidad. Como conclusión, los atletas y paraatletas participantes en el estudio presentan un perfil psicológico similar, no existiendo ninguna diferencia entre los grupos.
\end{abstract}

Palabras clave: Variables psicológicas; triatlón; paratriatlón; atletas de élite, CPRD.

Abstract. Just as has been reported in some studies, the psychological skills of para-athletes can be more consistent than those of athletes due to the overcoming of physical, communicative, and economic barriers, as well as traumas. The purpose of this study was to understand the differences in the psychological variables involved in sports performance between disabled and non-disabled triathlon athletes. 18 triathletes and 18 disabled triathletes of national and international level participated in the study. The questionnaire Características psicológicas relacionadas con el Rendimiento Deportivo (CPRD) was used in the study. The results obtained with the selected sample of expert triathletes indicate that there are no differences ( $p$ $<.05)$ in the psychological variables analyzed by the CPRD based on the presence or not of a disability. As a summary, athletes and para-athletes participating in the study have a similar psychological profile, no difference being found between the groups.

Key words: Psychological variables; triathlon; paratriathlon; elite athletes, CPRD.

\section{Introducción}

En el deporte de competición el uso de una buena estrategia psicológica permite al deportista rendir a niveles superiores (García-Naveira \& Díaz-Morales, 2010; Nicholls \& Polman, 2007; Olmedilla et al., 2015). Por este motivo, numerosos estudios sobre pericia deportiva se han centrado en los factores psicológicos que influyen negativa o positivamente en el rendimiento deportivo (Gimeno, Buceta, \& PérezLlantada, 2001; Mahamud, Tuero, \& Márquez, 2007). Algunos estudios que han analizado los factores que afectan al rendimiento deportivo desde la perspectiva psicológica coinciden en que la motivación, la autoconfianza, el estrés, la ansiedad, el establecimiento de metas y la concentración son los factores más importantes (Berengüí et al., 2013; RuizTendero \& Salinero-Martin, 2012). Además, reforzar estos elemento supon una optimización del rendimiento del deportista (García-Naveira \& Ruiz-Barquín, 2015).Se observa que mayoritariamente las investigaciones con este objeto de estudio han utilizado muestras de deportistas sin discapacidad. Sin embargo, el hecho de que en la actualidad esté creciendo el interés por los Juegos Paralímpicos, con la consiguiente inversión para que los atletas discapacitados (para-atletas) puedan asistir a este tipo de competiciones mejor preparados, está provocando que cada vez sea más importante conocer cómo los para-atletas pueden optimizar su rendimiento (Jefferies, Gallagher, \& Dunne, 2012). Los estudios de psicología con para-atletas comenzaron a adquirir importancia a partir de los años 90, una de las líneas de investigación ha sido la comparación entre atletas y para-atletas en varios parámetros psicológicos (Blumenstein \& Orbach, 2015). En este sentido, es posible pensar que los para-atletas debido a su condición y contexto puede que hayan desarrollado de manera consistente una serie de estrategias psicológicas que favorezcan el rendimiento deportivo (Torralba, Braz-Vieira, \& Rubio, 2017), debido a la superación de traumas y barreras físicas, comunicativas y económicas (Iezzoni, 2009; Martin, Malone, \& Hilyer, 2011) llegando a tener incluso motivaciones más complejas (Asken, 1991). Investigaciones en determinadas modalidades deportivas que han hecho comparaciones entre atletas y para-atletas indican que no

Fecha recepción: 31-07-18. Fecha de aceptación: 13-12-18 Guillermo Gomez-Marcos

guillermo.gomez06@alu.umh.es existen grandes diferencias en relación a las variables psicológicas relacionadas con el rendimiento deportivo (Martin, 1999; Sherrill, 1999) como se puede ver en la tabla 1(página 91). Sin embargo, a diferencia de los atletas, los para-atletas tienen que hacer frente a barreras físicas, comunicativas y económicas, además de superar traumas por lo que se podría pensar que pudieran existir diferencias entre unos y otros (Iezzoni, 2009; Martin et al., 2011). Además según Martin et al. (2011) los paraatletas entrenan menos las habilidades psicológicas por lo que su rendimiento puede verse afectada.

Desde la perspectiva de las modalidades deportivas, si nos centramos en el triatlón, la mayoría de los estudios están relacionados con aspectos fisiológicos, existiendo pocas investigaciones donde se ponga de manifiesto la importancia de los factores psicológicos en el rendimiento (Gucciardi, Mahoney, Jalleh, Donovan, \& Parkes, 2012; Stoeber, Uphill, \& Hotham, 2009). Respecto al para-triatlón, variante del triatlón para deportistas discapacitados que compiten en diferentes categorías asignadas en función del impacto que tiene el impedimento en el triatlón (IPC, 2015; Mujika, Orbananos, \& Salazar, 2015), no existen estudios que pongan de manifiesto cuáles son las variables psicológicas más importantes en el rendimiento deportivo. Además de la falta de estudios que describan las variables psicológicas que afectan al rendimiento en el deporte del triatlón, también hay escasez de estudios que aporten información actualizada sobre posibles diferencias entre deportistas con y sin discapacidad. Por lo tanto, el primer objetivo de esta investigación ha sido analizar el perfil psicológico y las correlaciones entre las variables psicológicas que afectan al rendimiento deportivo que presentan los

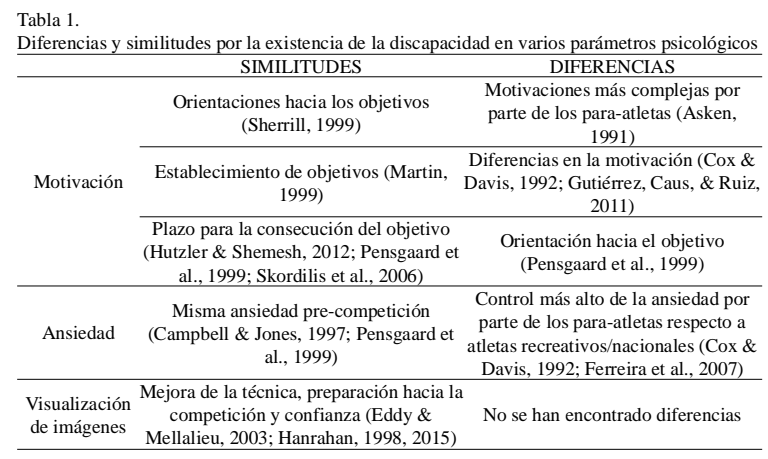


triatletas con y sin discapacidad de nivel nacional e internacional. El segundo objetivo ha sido conocer si hay diferencias en las variables psicológicas implicadas en el rendimiento deportivo por la existencia o no de la discapacidad.

\section{Método}

\section{Diseño}

Se trata de un estudio comparativo transversal, en la medida en que se establecen relaciones entre las variables examinando las diferencias que existen entre grupos en un momento temporal determinado (Ato, López, \& Benavente, 2013). El escaso tamaño de la muestra se debe a la poca participación el deporte del para-triatlon con los criterios de inclusión de la investigación, cumpliendo solo 32 para-triatletas en España con dichos criterios.

\section{Participantes}

18 triatletas y 18 para-triatletas (correspondientes a las seis categorías del para-triatlón) de nacionalidad española de alto nivel que cumplieron los siguientes criterios de inclusión: competir a nivel nacional, haber competido a nivel internacional al menos una vez y tener una experiencia mínima de tres años. El grupo triatlón se caracterizaba por tener 28,1 $\pm 5,4$ años, entrenar 19,8 \pm 6,3 horas a la semana y tener 9.0 \pm 5.0 años de experiencia. El grupo para-triatlón se caracterizaba por tener $40.2 \pm 9.3$ años, entrenar $18.0 \pm 6.8$ horas a la semana y tener 6.4 \pm 2.4 años de experiencia. Todos los participantes fueron informados de la finalidad de la investigación. El diseño y el desarrollo del estudio se realizaron respecto las recomendaciones éticas de la Declaración de Helsinki.

\section{Instrumento}

Para conocer las diferencias en las características psicológicas por la existencia o no de la discapacidad se utilizó el cuestionario «Cuestionario de Características Psicológicas Relacionadas con el Rendimiento Deportivo (CPRD). Esta escala fue desarrollada por Gimeno et al. (2001), y presenta confiabilidad y validez con una consistencia interna del cuestionario en su conjunto de 85 . El CPRD está compuesto por 55 ítems con un formato de respuesta tipo Likert con cinco puntos. Los 55 ítems forman parte de cinco variables: a) control del estrés, abarca las

\begin{tabular}{|c|c|c|c|c|c|c|c|}
\hline \multicolumn{8}{|c|}{ Para-triatlón } \\
\hline \multicolumn{2}{|c|}{ Variables } & $\mathrm{M}$ & SD & C & M & SD & $\mathrm{C}$ \\
\hline \multicolumn{2}{|c|}{$\begin{array}{l}\text { Control del Estrés } \\
\text { Influencia de la }\end{array}$} & 63.67 & 10.33 & 85 & 60.3 & 7.63 & 80 \\
\hline \multicolumn{2}{|c|}{$\begin{array}{l}\text { Influencia de la } \\
\text { Evaluación del }\end{array}$} & 38.11 & 7.34 & 95 & 35.0 & 6.99 & 95 \\
\hline \multirow{2}{*}{\multicolumn{2}{|c|}{$\begin{array}{l}\text { Motivación } \\
\text { Habilidad Mental }\end{array}$}} & 23.33 & 4.83 & 80 & 23.1 & 3.95 & 80 \\
\hline Habilidad Men & & 27.39 & 5.15 & 90 & 26.1 & 4.09 & 90 \\
\hline \multicolumn{8}{|c|}{ M = Media; SD = Desviación Típica; C = Centil } \\
\hline \multicolumn{8}{|c|}{ iables del CPRD en los triatletas y para-triatletas } \\
\hline \multicolumn{3}{|c|}{ Variables } & $\begin{array}{l}\text { Control I } \\
\text { del Estrés }\end{array}$ & $\begin{array}{r}\text { Influencia Evalua } \\
\text { Rendimiento }\end{array}$ & & Motivación & $\begin{array}{c}\begin{array}{c}\text { Habilidad } \\
\text { Mental }\end{array} \\
\text { Men }\end{array}$ \\
\hline \multirow{2}{*}{ Control Estrés - } & Triatlón & $\begin{array}{c}\text { CORR } \\
p\end{array}$ & 1 & $\begin{array}{l}.364 \\
.137 \\
\end{array}$ & & $\begin{array}{l}.061 \\
.809\end{array}$ & $\begin{array}{l}.481^{*} \\
.043 \\
\end{array}$ \\
\hline & Para-triatlón & $\begin{array}{c}\text { CORR } \\
p\end{array}$ & 1 & $\begin{array}{l}.497^{*} \\
.036\end{array}$ & & $\begin{array}{l}.383 \\
.117\end{array}$ & $\begin{array}{l}.576^{*} \\
.012\end{array}$ \\
\hline \multirow{4}{*}{$\begin{array}{c}\text { Influencia } \\
\text { Evaluación } \\
\text { Rendimiento }\end{array}$} & & CORR & .364 & 1 & & -.107 & .108 \\
\hline & Inlation & $p$ & 137 & & & .671 & .671 \\
\hline & Para-triatlón & corr & .137 & 1 & & .671 & .671 \\
\hline & & $p$ & 036 & & & .438 & 138 \\
\hline \multirow{3}{*}{ Motivación } & Triatlón & $\begin{array}{c}\text { corr } \\
p\end{array}$ & .061 & $\begin{array}{l}-.107 \\
.672\end{array}$ & & 1 & $.624^{*}$ \\
\hline & & corr & .383 & .195 & & 1 & .438 \\
\hline & Pard-triation & $p$ & .117 & .438 & & 1 & .069 \\
\hline \multirow{3}{*}{$\begin{array}{l}\text { Habilidad } \\
\text { Mental }\end{array}$} & Triatlón & corr & $481^{*}$ & .108 & & $.624^{*}$ & 1 \\
\hline & & corr & $.576^{*}$ & .364 & & .438 & \\
\hline & Para-triatlón & $p$ & .012 & .138 & & .069 & 1 \\
\hline \multicolumn{8}{|c|}{ Corr $=$ Correlación; $p=$ Significación $(\mathrm{p}<$.05); $*$ = Diferencias significativas } \\
\hline \multicolumn{8}{|c|}{ Tabla 4.} \\
\hline \multicolumn{8}{|c|}{ Diferencias en las variables del CPRD entre los triatletas y para-triatletas } \\
\hline \multicolumn{4}{|c|}{ Variables } & \\
\hline & Control del e & strés & & \multicolumn{4}{|c|}{.166} \\
\hline \multicolumn{4}{|c|}{ Influencia de la evaluación del rendimiento } & \multirow{2}{*}{\multicolumn{4}{|c|}{$\begin{array}{r}.079 \\
963\end{array}$}} \\
\hline \multicolumn{4}{|c|}{ Motivación } & & & & \\
\hline \multicolumn{4}{|c|}{ Habilidad Mental } & \multicolumn{4}{|c|}{.402} \\
\hline
\end{tabular}

características de la respuesta del deportista en relación con las demandas del entrenamiento y la competición (e.g. concentración) y las situaciones potencialmente estresantes que pueden provocar estrés y donde es necesario la presencia de control; b) influencia de la evaluación del rendimiento, abarca las características de la respuesta del deportista ante situaciones en las que éste u otras personas evalúan su rendimiento (e.g. perder la concentración); c) motivación, abarca la motivación por superarse día a día, el establecimiento de metas y la importancia del deporte en relación con otras actividades; d) habilidad mental, incluye habilidades psicológicas para favorecer el rendimiento deportivo (e.g. establecimiento de objetivos) y e) cohesión de equipo, la integración del deportista en su equipo o grupo deportivo (Gimeno et al., 2001) .

\section{Análisis de los datos}

Para conocer los datos de la muestra se realizó un análisis descriptivo (media y desviación típica). Se aplicaron las pruebas de normalidad Shapiro-Wilk y Kolmogorov-Smirnov para comprobarla normalidad de las variables del cuestionario. Posteriormente, para conocer las diferencias entre los grupos se ejecutó la prueba $T$ para muestras independientes en aquellas variables con distribución paramétrica y la U de Mann-Withney en las variables no paramétricas. Para analizar las correlaciones entre las variables del cuestionario se utilizó el coeficiente bivariado de Pearson. Se estableció un nivel de significación de $p<.05$. El análisis estadístico se realizó usando el Paquete Estadístico para Ciencias Sociales SPSS para Windows v.20.0.

\section{Resultados}

En la tabla 2 (página 9) se pueden ver los resultados de las diferentes variables que componen el cuestionario CPRD. Las puntuaciones en todas las variables analizadas son muy similares en los dos grupos estudiados. El grupo triatlón presenta valores un poco más elevados en las cuatro variables. Pese a los valores más altos, los dos grupos coinciden en los mismos centiles, excepto en la variable control del estrés, donde el grupo triatlón presenta el centil 85 y el grupo para-triatlón el centil 80 .

La tabla 3 (página 10) muestra las correlaciones entre las variables en el grupo de los triatletas y los para-triatletas. Como se puede observar, en los dos grupos existen correlaciones de medias altas entre las variables habilidad mental y control del estrés (triatlón: $\mathrm{r}=.48$; á $=.04$ y para-triatlón: $\mathrm{r}=.57$; á $=.01$ ). En el grupo triatlón también destaca la correlación entre habilidad mental y motivación ( $\mathrm{r}=.62$; á = .006). Por otro lado, en el grupo para-triatlón destaca la correlación entre control del estrés e influencia de la evaluación en el rendimiento ( $\mathrm{r}=.49$; $\mathrm{a}=$ =.03).

La tabla 4 (página 11) recoge los resultados correspondientes a las comparaciones entre los dos grupos estudiados respecto a las variables del CPRD. Se puede observar que no existen diferencias significativas entre las categorías en ninguna de las variables $(p<.05)$.

\section{Discusión}

Tal y como sugieren algunos estudios, se puede pensar que las habilidades psicológicas de los para-atletas pueden ser más potentes (Torralba et al., 2017), debido a la superación de barreras físicas, comunicativas y económicas, además de traumas (Iezzoni, 2009; Martin et al., 2011). Para profundizar sobre ello se precisa aumentar las investigaciones que caractericen los factores de rendimiento psicológico asociados con la discapacidad y el deporte (Blumenstein \& Orbach, 2015; Hanrahan, 1998, 2015).

En la tabla 5 (página 11) se puede observar que las puntuaciones y los centiles del presente estudio se corresponden con las más altas encontradas en investigaciones similares (Olmedilla, Ortega, Andreu, \& Ortín, 2010; Olmedilla et al., 2015) respecto a los baremos establecidos por Gimeno (2010).

Las similitudes con los jugadores profesionales de balonmano pueden ser debido al nivel de experiencia de sus jugadores (Chamberlain \& Coelho, 1993; García-Coll, Ruiz, \& Graupera, 2009). Respecto a los 


\begin{tabular}{|c|c|c|c|c|c|c|c|c|}
\hline \multirow[t]{2}{*}{ Variables } & \multicolumn{2}{|c|}{$\begin{array}{l}\text { Control del } \\
\text { estrés }\end{array}$} & \multicolumn{2}{|c|}{$\begin{array}{c}\text { Influencia de la } \\
\text { evaluación del } \\
\text { rendimiento }\end{array}$} & \multicolumn{2}{|c|}{ Motivación } & \multicolumn{2}{|c|}{$\begin{array}{c}\text { Habilidad } \\
\text { mental }\end{array}$} \\
\hline & PT & C & PT & C & PT & C & PT & C \\
\hline $\begin{array}{l}\text { Piragüistas categorías menores } \\
\text { (Mahamud et al., 2007) }\end{array}$ & 47.13 & 50 & 25.30 & 50 & 19.86 & 50 & 21.30 & 55 \\
\hline $\begin{array}{c}\text { Piragüistas senior } \\
\text { (Mahamud et al., 2007) } \\
\end{array}$ & 45.00 & 45 & 27.00 & 65 & 16.30 & 30 & 24.10 & 80 \\
\hline $\begin{array}{l}\text { Nadadores categorías menores } \\
\text { (Mahamud et al., 2007) }\end{array}$ & 38.35 & 25 & 24.00 & 50 & 19.40 & 50 & 20.55 & 45 \\
\hline $\begin{array}{l}\text { Futbolistas promesas equipo profesnioal } \\
\text { (Olmedilla et al., 2010) }\end{array}$ & l 62.75 & 85 & 33.69 & 85 & 25.50 & 85 & 23.31 & 70 \\
\hline $\begin{array}{c}\text { Jugadores Balonmano Profesionales } \\
\text { (Olmedilla et al., 2015) }\end{array}$ & 56.89 & 75 & 32.58 & 80 & 19.86 & 50 & 22.60 & 60 \\
\hline $\begin{array}{l}\text { Futbolistas categorías menores chilenos } \\
\text { (Lavarello, 2004) }\end{array}$ & 53.48 & 65 & 25.92 & 50 & 25.62 & 50 & 23.74 & 70 \\
\hline $\begin{array}{c}\text { Triatletas españoles } \\
\text { nacionales/internacionales }\end{array}$ & 63.67 & 85 & 38.11 & 95 & 23.33 & 80 & 27.39 & 90 \\
\hline $\begin{array}{c}\text { Para-triatletas españoles } \\
\text { nacionales/internacionales }\end{array}$ & 60.39 & 80 & 38.09 & 95 & 23.17 & 80 & 26.17 & 90 \\
\hline
\end{tabular}

jugadores promesas de futbol, la orientación al profesionalismo y los entrenamientos específicos pueden explicar las similares puntuaciones obtenidas (Olmedilla et al., 2010).

Sin embargo, otras investigaciones difieren en los perfiles psicológicos. Las puntuaciones en las variables del CPRD de los nadadores de categorías menores y los piragüistas senior y de categorías menores (Mahamud et al., 2007) y de los futbolistas de categorías menores (Lavarello, 2004) se diferencian de los resultados del presente estudio, obteniendo puntuaciones más bajas en todas las variables observadas. La causa de estas diferencias puede ser consecuencia del inferior nivel de pericia y el distinto nivel de competición. Como indican los estudios previos, cuanto mayor es el nivel de los deportistas, mayores son los valores que presentan (Chamberlain \& Coelho, 1993; García-Coll et al., 2009).

Respecto a las correlaciones entre las variables psicológicas del CPRD, ambos grupos muestran una correlación entre habilidad mental y control del estrés lo que pone de manifiesto la estrecha relación existente entre las habilidades mentales y la capacidad de controlar las situaciones estresantes, así como la capacidad de prevenir ansiedad gracias al control del estrés. Este resultado está apoyado en la investigación realizada por González-Campos, Valdivia-Moral, Cachón-Zagalaz, Zurita-Ortega, and Romero (2017) con futbolistas semiprofesionales. Los resultados difieren de las investigaciones realizadas con futbolistas jóvenes chilenos (Lavarello, 2004), y con bailarines amateur deportivos (Cantón \& Checa, 2012). Esta diferencia puede ser explicada por el diferente nivel de pericia presentado entre los diferentes estudios, siendo mayor el nivel de competición y pericia en los triatletas y paratriatletas respecto a los futbolistas y bailarines (Chamberlain \& Coelho, 1993; García-Coll et al., 2009). El grupo triatlón presenta una correlación entre motivación y habilidad mental, resultados que coinciden con el estudio realizado por con futbolistas jóvenes chilenos (Lavarello, 2004) y están en contraposición con el estudio realizado con bailarines deportivos de Cantón and Checa (2012). Respecto al grupo para-triatlón presenta una correlación entre el control del estrés y la influencia de la evaluación en el rendimiento. Otros estudios en la misma línea han obtenido resultados similares (Cantón \& Checa, 2012; Lavarello, 2004). Para Gimeno et al. (2001) se trata de dos variables con relación directa por la variable influencia de la evaluación en el rendimiento está relacionada con el estrés, aunque constituya un factor específico relacionado con la ansiedad social y con la interpretación inapropiada que el deportista realiza sobre su rendimiento.

Los resultados del presente estudio indican que no existen diferencias significativas en las variables psicológicas implicadas en el rendimiento deportivo que analiza el CPRD. Investigaciones anteriores encontraron resultados similares en las comparaciones entre atletas y para-atletas (DePauw \& Gavron, 2005). Los resultados obtenidos en la variable motivación y su relación con el rendimiento en atletas y paraatletas sigue la misma línea de otros estudios llevados a cabo con jugadores de baloncesto de alto nivel con y sin discapacidad (Hutzler \& Shemesh, 2012; Skordilis, Skafida, Chrysagis, \& Nikitaras, 2006) y con atletas y para-atletas noruegos de deportes de invierno (Pensgaard,
Roberts, \& Ursin, 1999). Respecto a la relación estrés y rendimiento y la comparación entre la existencia o no de la discapacidad, los resultados concuerdan con el estudio realizado con para-atletas en sillas de ruedas y con para-atletas noruegos de deportes de invierno (Pensgaard et al., 1999). Sin embargo, otros estudios difieren de los hallazgos encontrados, (Cox \& Davis, 1992) mostraron en su estudio una comparación entre para-atletas internacionales en silla de ruedas con atletas recreativos donde se encontraron diferencias en las habilidades psicológicas relacionadas con el rendimiento deportivo, obteniendo mayor grado los para-atletas (Cox \& Davis, 1992) y Ferreira, Chatzisarantis, Gaspar, and Campos (2007) en su estudio con atletas de nivel nacional y paraatletas internacionales encontraron una diferencia particular en la ansiedad previa a la competición, siendo mayor en los atletas que en los paraatletas. Los resultados obtenidos respecto a la habilidad mental y la visualización de imágenes, es similar a otros estudios con atletas con discapacidad física (Hanrahan, 1998, 2015) y discapacidad visual (Eddy \& Mellalieu, 2003). La experiencia deportiva y el nivel de competición similar de los atletas y para-atletas estudiados en las investigaciones puede ser el motivo de los resultados obtenidos.

\section{Conclusiones}

Una vez realizado el estudio y en base a los objetivos planteados se puede concluir a) que las puntuaciones altas en las variables del CPRD corresponden a perfiles psicológicos adecuados al nivel de la muestra seleccionada, b) que no existen diferencias en las variables psicológicas analizadas por el CPRD debido a la existencia o no de la discapacidad. La mayoría de los estudios analizados para la investigación no son actuales por lo que una futura línea de investigación interesante sería repetir los estudios y ver si se encuentran más diferencias entre grupos por la existencia o no de la discapacidad en el deporte de élite.

Como limitaciones del estudio se puede citar el escaso tamaño de la muestra. Al tratarse el para-triatlón de un deporte emergente, solo 32 para-triatletas españoles cumplen los criterios de inclusión presentados del estudio.

\section{Aplicaciones prácticas}

Como punto principal de las aplicaciones prácticas se puede destacar la necesidad de planificar, entrenar y medir las variables psicológicas. Para realizar ese trabajo herramientas como el CPRD son muy interesantes para ver el estado psicológico de los deportistas y detectar sus carencias. Por otro lado, a pesar de que la muestra seleccionada obtuvo valores óptimos en todas las variables estudiadas, es necesario mejorar desde el punto de vista psicológico por la incidencia que tiene en los resultados deportivos. Por último, gracias a los estudios previos y a los resultados de nuestra investigación y conociendo la estrecha relación existente entre las habilidades mentales y el estrés, un buen entrenamiento de estas habilidades mejoraría la preparación hacia la competición.

\section{Referencias}

Asken, M. J. (1991). The challenge of the physically challenged: Delivering sport psychology services to physically disabled athletes. The sport psychologist, 5(4), 370-381. doi: 10.1123/tsp.5.4.370.

Ato, M., López, J. J., \& Benavente, A. (2013). Un sistema de clasificación de los diseños de investigación en psicología. Anales de psicología, 29(3), 1038-1059. doi: 10.6018/analesps.29.3.178511

Berengüí, R., García-Pallarés, J., López-Gullón, J. M., Garcés de Los Fayos, E. J., Caravaca, E. C., \& Martinez- Abellán, A. (2013). Habilidades psicológicas fundamentales en las Luchas Olímpicas. Cuadernos de Psicología del Deporte, 12(2), 19-22.

Blumenstein, B., \& Orbach, I. (2015). Psychological Preparation for Paralympic Athletes: A Preliminary Study. Adapt Phys Activ Q, 32(3), 241-255. doi: 10.1123/apaq.2014-0235

Campbell, E., \& Jones, G. (1997). Precompetition anxiety and self- 
confidence in wheelchair sport participants. Adapted Physical Activity Quarterly, 14(2), 95-107. doi: 10.1123/apaq.14.2.95

Cantón, E., \& Checa, I. (2012). Medición de variables psicológicas en deportistas de baile deportivo. Universitas Psychologica, 11(3).

Cox, R. H., \& Davis, R. (1992). Pyschological skills of elite wheelchair athletes. Palaestra, 8(3), 16-21.

Chamberlain, C. J., \& Coelho, A. J. (1993). The perceptual side of action: Decision-making in sport. Advances in psychology (Vol. 102, pp. 135-157): Elsevier.

DePauw, K. P., \& Gavron, S. J. (2005). Disability sport (2a ed.). Leeds: Human Kinetics.

Eddy, K. A., \& Mellalieu, S. D. (2003). Mental imagery in athletes with visual impairments. Adapted Physical Activity Quarterly, 20(4), 347-368. doi: 10.1590/S1413-35552012005000123

Ferreira, J. P., Chatzisarantis, N., Gaspar, P. M., \& Campos, M. J. (2007). Precompetitive anxiety and self-confidence in athletes with disability. Percept Mot Skills, 105(1), 339-346. doi: 10.2466/ pms.105.1.339-346

García-Coll, V., Ruiz,L. M., \& Graupera, J.L.(2009). Perfiles decisionales de jugadores y jugadoras de voleibol de diferente nivel de pericia. RICYDE. Revista Internacional de Ciencias del Deporte, 14(5), 123-137. doi: 10.5232/ricyde2009.01410

García-Naveira, A., \& Díaz-Morales, J. F. (2010). Relación entre optimismo/pesimismo disposicional, rendimiento y edad en jugadores de fútbol de competición. Revista Iberoamericana de Psicología del Ejercicio y el Deporte VOL. VN $N^{\circ}$ 1, 5(1), 45.

García-Naveira, A., \& Ruiz-Barquín, R. (2015).ADAPTACIÓN DEL ISRA-BA LA POBLACIÓN DEPORTIVA A TRAVÉS DE JUGADORES DE FÚTBOL DE RENDIMIENTO. Ansiedad y Estrés, 21(1).

Gimeno, F. (2010). El cuestionario «Características Psicológicas relacionadas con el Rendimiento Deportivo (CPRD): Características psicométricas. Experiencias en Psicología del Deporte. Madrid: Dykinson.

Gimeno, F., Buceta, J. M., \& Pérez-Llantada, M. C. (2001). El cuestionario «características psicológicas relacionadas con el rendimiento deportivo»(CPRD): Características psicométricas. Análise Psicológica, 19(1), 93-113. doi: 10.14417/ap.346

González-Campos, G., Valdivia-Moral, P., Cachón-Zagalaz, J., ZuritaOrtega, F., \& Romero, O. (2017). Influencia del control del estrés en el rendimiento deportivo: la autoconfianza, la ansiedad y la concentración en deportistas. RETOS. Nuevas Tendencias en Educación Física, Deporte y Recreación(32).

Gucciardi, D. F., Mahoney, J., Jalleh, G., Donovan, R. J., \& Parkes, J. (2012). Perfectionistic profiles among elite athletes and differences in their motivational orientations. J Sport Exerc Psychol, 34(2), 159-183. doi: 10.1123/jsep.34.2.159

Gutiérrez, M., Caus, N., \& Ruiz, L.-M.(2011). The influence of parents on achievement orientation and motivation for sport of adolescent athletes with and without disabilities. Journal of Leisure Research, 43(3), 355-382. doi: 10.1080/00222216.2011.11950241

Hanrahan, S. J.(1998). Practical considerations for working with athletes with disabilities. The Sport Psychologist, 12(3), 346-357. doi: 10.1123/tsp.12.3.346

Hanrahan, S. J. (2015). Psychological Skills Training for Athletes With Disabilities. Australian Psychologist, 50(2), 102-105. doi: 10.1111/ ap.12083

Hutzler,Y., \& Shemesh, R. (2012). Self-efficacy, task and ego orientation, and family support in wheelchair and able-bodied basketball players. Therapeutic Recreation Journal, 46(2), 73-90.

Iezzoni, L. I. (2009). Public health goals for persons with disabilities: looking ahead to 2020. Disabil Health J, 2(3),111-115. doi: 10.1016/ j.dhjo.2009.03.002

IPC. (2015). Explanatory guide to Paralympic Classification. Paralympic summer sports. International Paralympic Committee. from https:/ /www.paralympic.org/sites/default/files/document/

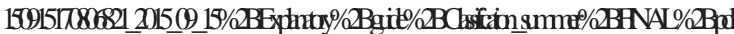

Jefferies, P., Gallagher, P., \& Dunne, S. (2012). The Paralympic athlete: a systematic review of the psychosocial literature. Prosthet Orthot Int, 36(3), 278-289. doi: 10.1177/0309364612450184

Lavarello, J. (2004). Estudio correlacional entre las variables medidas a través del CPRD en futbolistas de las divisiones menores. Revista de Medicina del deporte Cubana, 3, 18-26.

Mahamud, J., Tuero, C., \& Márquez, S. (2007). Características psicológicas relacionadas con el rendimiento: comparación ente los requerimientos de los entrenadores y la percepción de los deportistas. Revista de Psicología del deporte, 14(2), 0237-0251.

Martin, J. J. (1999). A personal development model of sport psychology for athletes with disabilities. Journal of Applied Sport Psychology, 11(2), 181-193. doi: 10.1080/10413209908404199.

Martin, J. J., Malone, L. A., \& Hilyer, J. C. (2011). Personality and mood in women's Paralympic basketball champions. Journal of Clinical Sport Psychology, 5(3), 197-210. doi: 10.1123/jcsp.5.3.197

Mujika, I., Orbananos, J., \& Salazar, H. (2015). Physiology and training of a world-champion paratriathlete. Int J Sports Physiol Perform, 10(7), 927-930. doi: 10.1123/ijspp.2014-0487

Nicholls, A. R., \& Polman, R. C. (2007). Coping in sport:A systematic review. J Sports Sci, 25(1), 11-31. doi: 10.1080/ 02640410600630654

Olmedilla, A., Ortega, E., Andreu, M. D., \& Ortín, F. J. (2010). Programa de intervención psicológica en futbolistas: evaluación de habilidades psicológicas mediante el CPRD. Revista de Psicología del Deporte, 19(2), 0249-0262.

Olmedilla, A., Ortega, E., Garcés de los Fayos, E., Abenza, L., Blas, A., \& Laguna, M. (2015). Perfil psicológico de los jugadores profesionales de balonmano y diferencias entre puestos específicos. Revista Latinoamericana de Psicología, 47(3), 177-184. doi: 10.1016/ j.rlp.2015.06.005

Pensgaard, A. M., Roberts, G. C., \& Ursin, H. (1999). Motivational Factors and Coping Strategies of Norwegian Paralympic and Olympic Winter SportAthletes. Adapted Physical Activity Quarterly, 16(3), 238-250. doi: 10.1123/apaq.16.3.238

Ruiz-Tendero, G., \& Salinero-Martin, J. J. (2012). Psycho-social factors determining success in high-performance triathlon: compared perception in the coach-athlete pair. Percept Mot Skills, 115(3), 865-880. doi: 10.2466/08.25.pms.115.6.865-880

Sherrill, C. (1999). Disability sport and classification theory: Anew era. Adapted Physical Activity Quarterly, 16(3), 206-215. doi:10.1123/ apaq.16.3.206

Skordilis, E. K., Skafida, F. A., Chrysagis, N., \& Nikitaras, N. (2006). Comparison of sport achievement orientation of male wheelchair basketball athletes with congenital and acquired disabilities. Percept Mot Skills, 103(3), 726-732. doi: 10.2466/pms.103.3.726-732

Stoeber, J., Uphill, M. A., \& Hotham, S. (2009). Predicting race performance in triathlon: the role of perfectionism, achievement goals, and personal goal setting. J Sport Exerc Psychol, 31(2), 211-245. doi: 10.1123/jsep.31.2.211

Torralba, M. Á., Braz-Vieira, M., \& Rubio, M. J. (2017). Motivos de la práctica deportiva de atletas paralímpicos españoles. Revista de psicología del deporte, 26(1), 49-60.

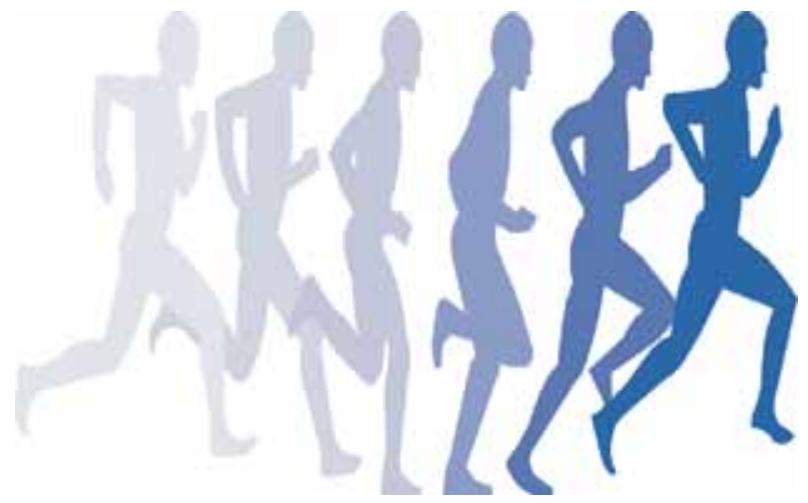

